\title{
GaN Nanowires Synthesized by Electroless Etching Method
}

\author{
A. Najar ${ }^{1}$, A. B. Slimane ${ }^{1}$, D. H. Anjum ${ }^{2}$, T. K. $\mathrm{Ng}^{1}$ and B. S. Ooi ${ }^{*}$ \\ ${ }^{I}$ Photonics Laboratory, King Abdullah University of Science and Technology (KAUST), Kingdom of Saudi Arabia \\ ${ }^{2}$ Advanced Nanofabrication, Imaging and Characterization Core Facilities, King Abdullah University of Science and Technology (KAUST), \\ Kingdom of Saudi Arabia \\ *E-mail : boon.ooi@kaust.edu.sa
}

\begin{abstract}
Ultra-long Gallium Nitride Nanowires is synthesized via metal-electroless etching method. The morphologies and optical properties of GaN NWs show a single crystal GaN with hexagonal Wurtzite structure and high luminescence properties.
\end{abstract}

OCIS codes:

\section{Introduction}

Nanowires (NWs) have a large surface-to-volume ratio and can also exhibit a reduced dimensionality [1]. In addition, compared to quantum dots, NWs are easier to contact and to handle and they can be used as active devices, as well as interconnects or waveguides [2, 3]. Furthermore, their small cross-sections can accommodate much larger lattice mismatch and thermal expansion difference compared to planar layers, because strain is released at the free NW sidewalls [4]. The design and fabrication of many different types of devices have already been demonstrated for applications in electronics, computing, photonics, sensing, and biology [3,5]. Gallium nitride (GaN) is a robust wideband- gap semiconductor with a high melting point, high carrier mobility, and high electrical breakdown field, it is a prime candidate for use in future high-performance, high power optoelectronic devices [6]. Single-crystalline gallium nitride nanowires and nanotubes show promise for realizing photonic and biological nanodevices such as blue-light emitting diodes (LEDs), short-wavelength ultraviolet nanolasers [7,8], and nanofluidic biochemical sensors. Virtually all reported synthetic schemes for GaN-based nanowires to date have employed either laser ablation [8,9], metal-organic chemical vapor deposition (MOCVD) [10, 11], or molecular beam epitaxy (MBE) [12]. Here, we report for the first time the synthesis and characterizations of ultra-long $(>10 \mu \mathrm{m}) \mathrm{GaN}$ nanowires using a novel metal-electroless etching method.

\section{Experiments}

The unintentionally doped n-type GaN film used in this study was grown on c-axis (0001) sapphire substrate with resistivity lower than $0.05 \Omega . \mathrm{cm}$. The thickness of the GaN film was $30 \mu \mathrm{m}$, and the carrier concentration of the film was $3.4 \times 10^{17} \mathrm{~cm}^{-3}$. (we should not use 'unintentionally' doped if this is the doping level - please confirm) The GaN samples were cleaved and cleaned by sonication in acetone and then in 2-propanol for $5 \mathrm{~min}$ in each solution. Then the samples were immersed in $\mathrm{HNO}_{3}$ at $65{ }^{\circ} \mathrm{C}$ for $15 \mathrm{~min}$. After that the samples were rinsed in DI water and methanol. Narrow stripes of $10 \mathrm{~nm}$ thick Pt, separated by a few millimeters, were deposited on the GaN samples using a sputtering system. The samples were etched in the HF: $\mathrm{CH}_{3} \mathrm{OH}: \mathrm{H}_{2} \mathrm{O}_{2}(2: 1: 2)$ solution under UV illumination for $180 \mathrm{~min}$. Finally the samples were removed from the solution and rinsed with DI water. The surface morphology of the samples was investigated using scanning electron microscopy (SEM), transmission electron microscopy (TEM), and photoluminescence (PL).The measurements were performed at room temperature.

\section{Results and discussion}

Fig. 1(a) shows the scanning electron microscope (SEM) image of the sample synthesized with 180 min reaction time. The surface of the sample is covered with nanowires with length from 1 to $12 \mu \mathrm{m}$. SEM image shows that the sample's surface is clean with no particle impurities. A histogram of diameters as measured from the SEM image is shown in Fig 1(b). The nanowires have typical diameters from $17-50 \mathrm{~nm}$, and an average diameter of $33 \mathrm{~nm}$. Fig. 1(c) and (d) shows high resolution TEM images and the corresponding calculated Fast Fourier Transform (FFT) patterns of individual $\mathrm{GaN}$ nanowire, which matches the [0001] diffraction pattern. The analysis of the GaN NWs shows that the nanowires have a single crystalline, wurtzite structure. The measured interval of the closest 
interplanar distance is $0.25 \mathrm{~nm}$, which is corresponding to the crystal plane (100) of GaN, and is in accordance with the XRD result (results not shown).
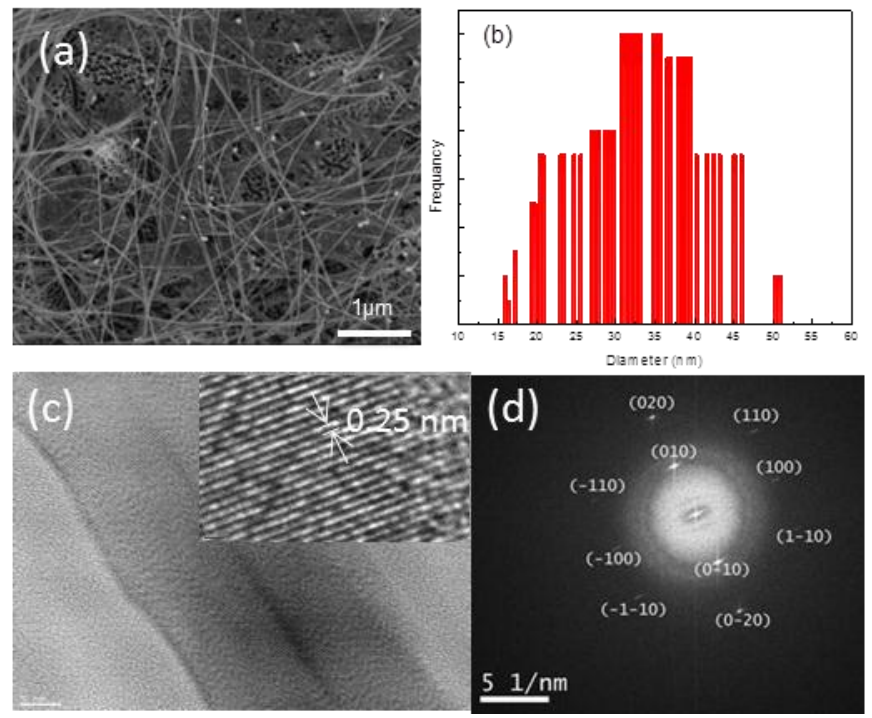

Fig. 1: (a) SEM image of the GaNNWs, (b) Histogram of diameters for GaNNWs,(c) TEM image of individual NW with HRTEM in the inset and (d) FFT image taken from image (b).

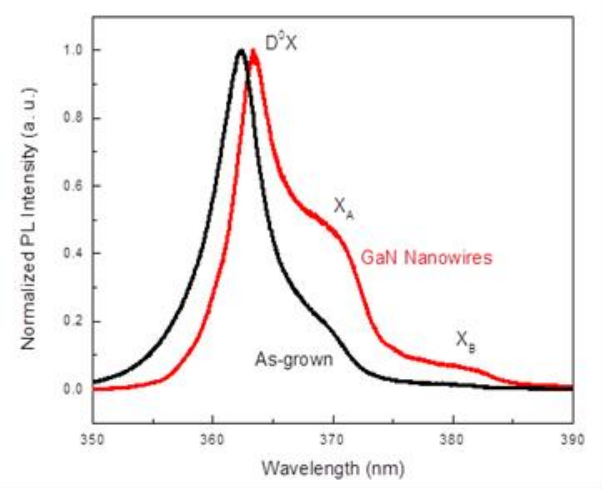

Fig. 2: PL spectra for the as-grown (black) and GaN NWs (red)

Fig. 2 gives the PL spectra of the initial GaN layer (black curve) and of the GaNNWs (red curve) at room temperature. The luminescence spectra of GaNWs are predominated by the $\mathrm{D}^{0}{ }_{\mathrm{X}}$ bound exciton, $\mathrm{X}_{\mathrm{A}}$ and $\mathrm{X}_{\mathrm{B}}$ excitonic emission. The positions of the $\mathrm{D}^{0}{ }_{\mathrm{X}}(\sim 364 \mathrm{~nm}), \mathrm{X}_{\mathrm{A}}(\sim 369.7 \mathrm{~nm})$ and $\mathrm{X}_{\mathrm{B}}(\sim 381.9 \mathrm{~nm})$ peaks in the luminescence spectrum of the GaNNWs and is shifted by $\sim 25 \mathrm{meV}$ towards lower energies comparing with the position of the corresponding excitonsin GaN layers. This means that a considerable strain remains in the GaN NWs. In our experiments, the nanowires formation mixed with porous $\mathrm{GaN}$ formed in the surface reduced the lattice constraint imposed by the underlying substrate and strained GaN closer to the substrate, and hence relieved the biaxial tensile stress and the corresponding uni-axial compressive stress. This argument is consistent with the observation of the red-shift in the peak PL wavelength. We believe that the ability to use inexpensive, large area, and process compatible sapphire wafers for the synthesize of GaN NWs, single crystalline and without the use of templates represents an important step towards realizing devices based on lateral or vertical integrated III-nitride nanowires.

\section{Conclusion}

Single-crystal GaN nanowires were synthesized successfully via Pt electroless etching method. The GaN NWs structure was studied by SEM, HRTEM and PL. The results showed that the nanowires are pure hexagonal wurtzite single-crystal $\mathrm{GaN}$ with lengths for 1 to $12 \mu \mathrm{m}$ and diameters from $17-50 \mathrm{~nm}$, and give strong PL emission at $364 \mathrm{~nm}$. This GaN NWs can potentially find applications in novel nano-electronic and photonic devices.

\section{References}

[1] L. Cademartiri and G. A. Ozin. "Ultrathin Nanowires", Adv. Mater., 21, 1013, (2008).

[2] C. M. Lieber. "Building a big future from small things", MRS Bulletin, 28, 486, (2003).

[3] P. J. Pauzauskie and P. Yang. "Nanowire photonics", Materials Today, 9, 36, (2006).

[4] F. Glas. "Critical dimensions for the plastic relaxation of strained axial heterostructures in free-standing nanowires", Phys. Rev. B,

74:121302, (2006).

[5] C. M. Lieber and Z. L. Wang. "Functional Nanowires", MRS Bulletin, 32, 99, (2007).

[6] Johnson, J. C. et al "Single gallium nitride nanowire lasers", Nature Mater., 1, 106 - 110 (2002)

[7] Goldberger, J. et al "Single-crystal gallium nitride nanotubes", Nature 422, 599-602, (2003).

[8] Huang, Y.; Duan, X., Cui, Y., Lieber, C. M. "Gallium Nitride Nanowire Nanodevices", Nano Lett., 2, 101-104, (2002).

[9] Choi, H.; Johnson, J. et al, "Self-organized GaN quantum wire UV lasers", J. Phys. Chem. B, 107, 8721, (2003).

[10] T. Kuykendall et al, "Metalorganic Chemical Vapor Deposition Route to GaN Nanowires with Triangular Cross Sections", Nano Lett, 3 , 1063-1066, (2003).

[11] X. Weng et al, "Nature of Catalyst Particles and Growth Mechanisms of GaN Nanowires Grown by Ni-Assisted Metal-Organic Chemical Vapor Deposition", Nanotechnology, 20, 085610, (2009).

[12] K. A. Bertness et al, " Catalyst-Free Growth of GaN Nanowires", J. of Elect. Mat., 35, 4, (2006). 